\title{
Schirmwirkung von Hochfrequenz (HF)-Schutzkleidung: Untersuchung verschiedener Konstruktionsmerkmale
}

\author{
V. Arps and K. Scheibe \\ Fachhochschule Kiel, Fachbereich Informatik und Elektrotechnik, Grenzstr. 5, 24149 Kiel, Germany
}

Zusammenfassung. Die Messverfahren zur Bestimmung der Schutzwirkung von HF-Schutzkleidung sind in der Norm DIN 32780-100 festgelegt. Entsprechend diesen Anforderungen wird die elektrische und magnetische Schirmdämpfung bestimmt und daraus als Maß für die Schutzwirkung die elektromagnetische Schirmdämpfung berechnet. Diese ist eine der SAR vergleichbare Größe.

In diesem Beitrag werden die Einflüsse verschiedener Konstruktionsmerkmale von HF-Schutzanzügen auf die elektromagnetische Schirmdämpfung untersucht. $\mathrm{Zu}$ diesen gehören die nach MIL STD 285 vermessene elektrische Schirmdämpfung der verwendeten Gewebe. Weiter werden verschiedene Teilbereiche der HF-Schutzkleidung auf ihre Schutzwirkung untersucht. Der Schwerpunkt liegt hierbei auf der Fragestellung inwieweit Verschlüsse, Reißverschlüsse oder leitfähiges Klettband, die Schutzwirkung beeinträchtigen.

$\mathrm{Zu}$ diesem Zweck werden zwei Schutzanzüge unterschiedlicher Konstruktion vergleichend vermessen. Es handelt sich dabei um einen bereits im Handel befindlichen und entsprechend der Norm zertifizierten Anzug und einen neuen Prototyp, welcher nach verschiedenen Gesichtspunkten optimiert wurde. Schwachstellen der Konstruktion werden herausgearbeitet und Ansatzpunkte für weitere Verbesserungen erarbeitet.

The measuring methods for determining the shielding effectiveness of radiofrequency (RF)-protective clothing are defined in German Standard DIN 32780-100. According to this standard, both the electric and the magnetic shielding effectiveness are measured in order to calculate the electromagnetic shielding effectiveness. The electromagnetic shielding effectiveness is an adequate quality criterion for the degree of protection and also compares well with the Specific Absorption Rate (SAR).

In this article, the impact of different design features on the electromagnetic shielding effectiveness is analyzed. The electric shielding effectiveness of the used shielding materials is measured according to MIL STD 285 and thereupon taken into account. Moreover, different parts of the RF-Protective Clothing are analyzed in terms of shielding

Correspondence to: V. Arps

(verena.arps@sma.de) properties. The main focus is on the question to what extent seams and zippers impair the shielding properties. Therefore, two RF-protective suits with different design features are tested and compared. One of the tested suits is accredited according to German Standard and is already on the market. The other one is an optimized prototype. Weak spots concerning design features and starting points for further optimization are worked out.

\section{Einleitung}

Die Wärmewirkung hochfrequenter elektromagnetischer Felder auf den Organismus gilt im Wesentlichen als erforscht und ist daher maßgeblich für die Festlegung von Expositionsgrenzwerten. Die pro Zeiteinheit vom Gewebe aufgenommene Energie hängt von seiner spezifischen Wärmekonstante und der umgewandelten Leistung ab. Als Maß hierfür eignet sich die in der Einheit Watt pro Kilogramm angegebene Spezifische Absorptionsrate (SAR), mit der sich die in Wärme umgewandelte Leistung zu der Masse des exponierten Gewebes ins Verhältnis setzen lässt (Koch, 2001).

Es gilt:

$S A R=\frac{P}{m}=c * \frac{\Delta T}{\Delta t}$

(P: Absorbierte Leistung [W], m: Masse [kg], c: Spezifische Wärmekonstante [J/kg*K], T: Temperatur [K], t: Zeit [s])

Die Grenzwerte für berufliche Expositionen elektromagnetischer Felder werden in der Berufsgenossenschaftlichen Unfallverhütungsvorschrift BGV B11 vorgegeben. Bei der Festlegung der Expositionsgrenzwerte wird unterschieden zwischen den sog. "Basis-" Grenzwerten und den sog. "abgeleiteten" Grenzwerten. Die aufgrund der biologischen Wirkungen festgelegten Basisgrenzwerte, bei deren Einhaltung es nach dem heutigen Stand der Wissenschaft weder zu Beeinträchtigungen noch zu Schädigungen von Personen kommt, dürfen nicht überschritten werden. Der Basisgrenzwert des für die Deutsche Norm Schutzkleidung entscheidenden Frequenzbereiches $80 \mathrm{MHz}$ bis $1 \mathrm{GHz}$ ist in der Vorschrift als maximal zulässige SAR beschrieben. Da die SAR nur mit messtechnisch sehr aufwendigen Verfahren direkt gemessen werden kann, werden zusätzlich auch die 
abgeleiteten Grenzwerte genannt (Hummel und Koch, 2004). Diese Grenzwerte wurden aus den Basiswerten so ermittelt, dass diese selbst bei ungünstigsten Expositionsbedingungen eingehalten werden. Sie geben die maximale elektrische und magnetische Feldstärke an, der eine Person ausgesetzt werden darf.

\section{Schirmdämpfung bei HF-Schutzanzügen}

Die Schirmdämpfung ist grundlegend für die Schutzwirkung eines HF-Schutzanzuges und damit auch für die Ermittlung der Exposition des Trägers. Sie ist somit ein geeignetes Qualitätskriterium für Schutzkleidung.

Die Schirmdämpfung ist nach MIL STD 285 definiert als das logarithmische Verhältnis einer Signalgröße an einem ungeschirmten Messort zur Signalgröße an demselben Messort nach Einbringung eines Schirms (Koch, 2001). Die Signalgröße variiert mit der jeweiligen Anwendung und dem Frequenzbereich. Angewendet auf die SAR ergibt sich folgender Zusammenhang:

$S E_{S A R}=10 * \log \frac{S A R_{\text {ohne }}}{S A R_{\text {mit }}}$

( $\mathrm{SE}_{S A R}$ : SAR Schirmdämpfung [dB], $\mathrm{SAR}_{\text {ohne }}$ : Zeitlich gemittelte spezifische Absorptionsrate in einem Körpermodell ohne Schirmung [W/kg], SAR ${ }_{\text {mit }}$ : Zeitlich gemittelte spezifische Absorptionsrate in einem Körpermodell mit Schirmung $[\mathrm{W} / \mathrm{kg}])$

Die elektromagnetische Schirmdämpfung lässt sich wie folgt berechnen:

$$
\begin{aligned}
& S E_{\text {em }}=10 * \log \frac{2}{10^{-\frac{S E_{e}}{10}}+10^{-\frac{S E_{m}}{10}}}= \\
& 10 * \log \frac{2}{\left(\frac{E_{\text {mit }}}{E_{\text {ohne }}}\right)^{2}+\left(\frac{H_{\text {mit }}}{H_{\text {ohne }}}\right)^{2}}
\end{aligned}
$$

( $\mathrm{SE}_{e m}$ : Elektromagnetische Schirmdämpfung $[\mathrm{dB}]$ )

Diese Größe setzt sich zusammen aus der elektrischen Schirmdämpfung:

$S E_{e}=20 * \log \frac{E_{\text {ohne }}}{E_{\text {mit }}}$

( $\mathrm{SE}_{e}$ : Elektrische Schirmdämpfung $[\mathrm{dB}], \mathrm{E}_{\text {ohne }}$ : Effektivwert der elektrischen Feldstärke in einem Körpermodell ohne Schirmung $[\mathrm{V} / \mathrm{m}], \mathrm{E}_{m i t}$ : Effektivwert der elektrischen Feldstärke in einem Körpermodell mit Schirmung [V/m])

und der magnetischen Schirmdämpfung:

$S E_{m}=20 * \log \frac{H_{\text {ohne }}}{H_{\text {mit }}}$

( $\mathrm{SE}_{m}$ : Magnetische Schirmdämpfung [dB], $\mathrm{H}_{\text {ohne }}$ : Effektivwert der magnetischen Feldstärke in einem Körpermodell ohne Schirmung [A/m], $\mathrm{H}_{\text {mit }}$ : Effektivwert der magnetischen Feldstärke in einem Körpermodell mit Schirmung [A/m])

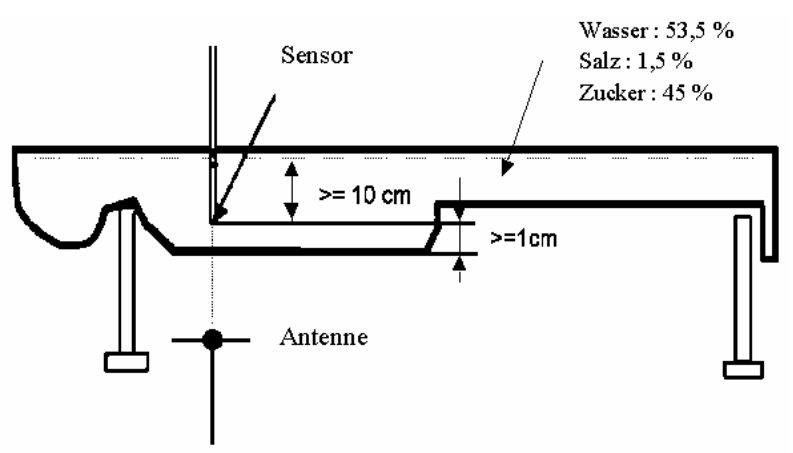

Abbildung 1. Prüfaufbau, SAR-Messverfahren.

\section{Mess- und Prüfverfahren}

Entsprechend der Norm DIN 32780-100 sind zwei unterschiedliche Prüfverfahren zulässig.

\subsection{SAR-Verfahren}

Bei dem SAR-Verfahren wird die SAR-Schirmdämpfung durch Messung der internen elektrischen Feldstärke bestimmt. Bild 1 zeigt den hierfür nach der Norm DIN 32780100 vorgeschriebenen Prüfaufbau.

Ein menschengroßes Körpermodell wird über einer Sendeantenne positioniert. Das Körpermodell ist mit einer muskeläquivalenten Flüssigkeit gefüllt, die zu den in Bild 1 angegebenen Anteilen aus Wasser, Zucker und Salz besteht. In die Flüssigkeit wird ein Feldsensor mindestens $10 \mathrm{~cm}$ tief eingeführt. Der Mindestabstand zum Boden des Körpermodells beträgt $1 \mathrm{~cm}$. Die Messungen werden mit und ohne Schutzkleidung jeweils nacheinander im Kopf- und im Brustbereich des Körpermodells durchgeführt. Hierbei werden zusätzlich verschiedene Positionen des Anzuges simuliert. Aufgrund des aufwändigen Prüfaufbaus wird dieses Verfahren in der Praxis jedoch kaum angewendet.

\subsection{Feldstärkemessverfahren}

Beim Feldstärkeverfahren dagegen wird die Schirmwirkung durch die elektromagnetische Schirmdämpfung, die mit Hilfe der Raummittelpunktmethode bestimmt wird, ausgedrückt. Bei Messungen nach dem Feldstärkemessverfahren wird die Tatsache genutzt, dass die elektromagnetische Schirmdämpfung eine mit der SAR vergleichbare Größe ist. Das Körpermodell dient bei diesem Prüfverfahren lediglich dazu, den Schutzanzug während der Messungen in Position zu halten. Es ist daher nicht nötig dieses mit einer gewebeäquivalenten Flüssigkeit zu füllen.

Um starke Hohlraumresonanzen im Innern der Schutzkleidung zu vermeiden wird das verwendete Körpermodell teilweise mit verlustbehaftetem Absorbermaterial gefüllt. Bild 2 zeigt den Prüfaufbau für die Messungen nach dem Feldstärkeverfahren. 


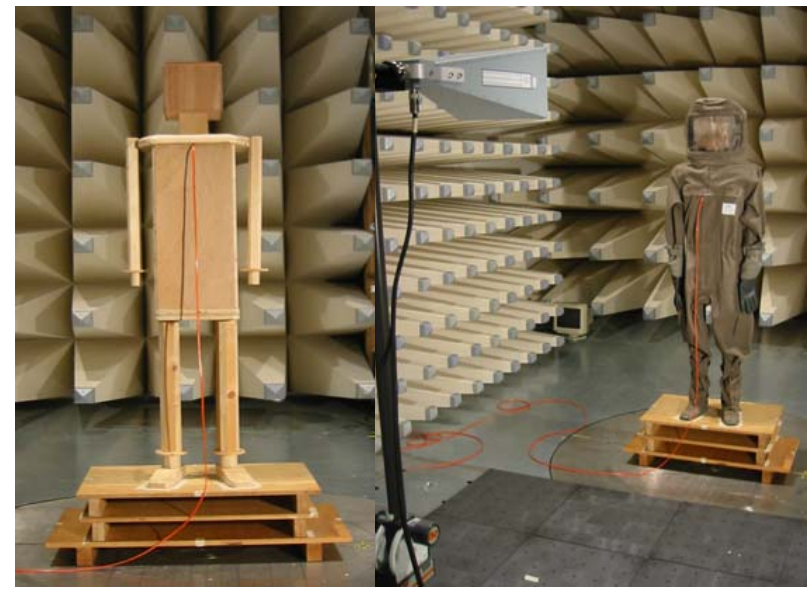

Abbildung 2. Prüfaufbau, Feldstärkemessverfahren.

Nach DIN 32780-100 ist es erforderlich, das Körpermodell, in dem sich die Empfängersonden befinden, mit einem Abstand von $0,8 \mathrm{~m}$ bis $3 \mathrm{~m}$ zu einer gegenüberstehenden Sendeantenne zu positionieren. Der Kopf und der Thorax des Körpermodells bilden jeweils einen Hohlkörper und bieten somit Platz die Messvorrichtung aufzunehmen. Die Messungen werden jeweils im Kopf- sowie im Brustbereich des Körpermodells bei sonst unveränderter Messanordnung jeweils mit und ohne Schutzanzug nacheinander einzeln durchgeführt. Das Körpermodell wird zur Durchführung der Messungen zunächst mit der Frontseite zur Antenne positioniert und dann in mindestens drei Schritten um seine Längsachse gedreht. Ziel ist es hierbei, Erkenntnisse über das Schirmdämpfungsverhalten einzelner Teilbereiche der HFSchutzkleidung, insbesondere Bereiche mit Verbunden und Verschlüssen, zu erlangen.

Als Ergebnis der Messung wird für jeden Frequenzschritt der kleinste der erhaltenen Werte für die elektromagnetische Schirmdämpfung ermittelt und angegeben.

\section{Ergebnisse}

Es werden zwei Schutzanzüge unterschiedlicher Konstruktion vergleichend vermessen. Es handelt sich dabei um einen bereits im Handel befindlichen und entsprechend der Norm zertifizierten Anzug und einen neuen Prototyp, welcher nach verschiedenen Gesichtspunkten optimiert wurde. Die Optimierung diente insbesondere dem verbesserten Tragekomfort der HF-Schutzkleidung.

Der Prototyp besteht aus einer Außenlage des bereits bei dem zertifizierten Schutzanzug verwendeten Gewebes PM 30 FR. Für die Innenlage wird jedoch statt des vorher verwendeten Gewebes PM 12-4 das neuartige Gewebe PCCS 2131 verwendet. Bild 3 zeigt die Ergebnisse der nach MIL STD 285 durchgeführten Schirmdämpfungsuntersuchung der Schirmgewebe.

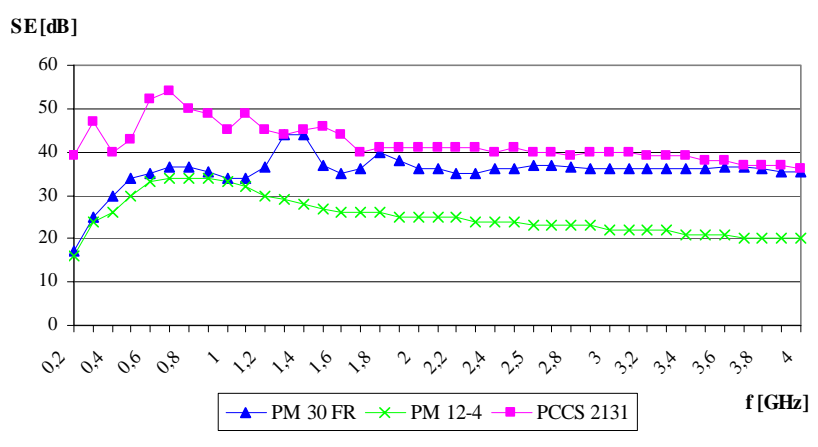

Abbildung 3. Schirmdämpfung der unterschiedlichen Schirmgewebe.
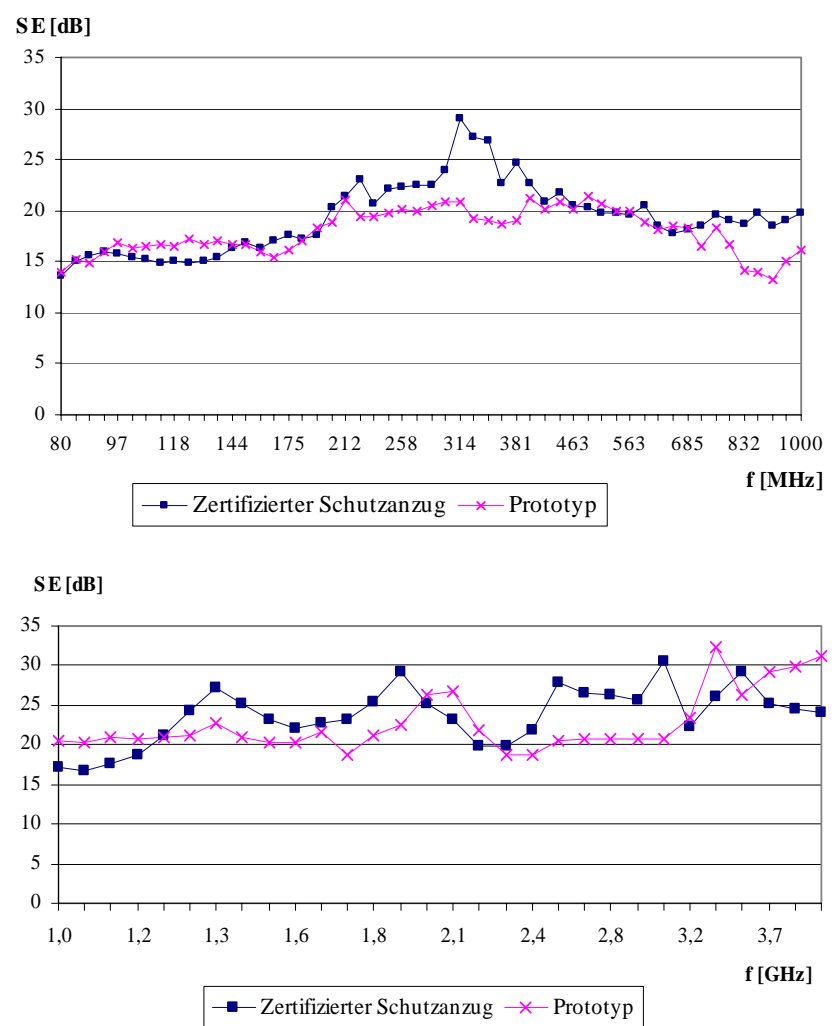

Abbildung 4. (A): Schirmdämpfung beider HF-Schutzanzüge (80 MHz-1 GHz), (B): Schirmdämpfung beider HF-Schutzanzüge $(1 \mathrm{GHz}-4 \mathrm{GHz})$

Bei den an den Schirmgeweben durchgeführten Schirmdämpfungsuntersuchungen zeigt sich, dass Gewebe PCCS 2131 im gesamten Frequenzverlauf eine deutlich bessere Schirmwirkung aufweist als das Gewebe PM 12-4.

Im Rahmen der durchgeführten Vergleichsuntersuchungen der zwei Schutzanzüge konnte jedoch festgestellt werden, dass der zertifizierte Schutzanzug und der verbesserte Prototyp ein fast identisches Schirmdämpfungsverhalten aufweisen (vgl. Bild 4). 


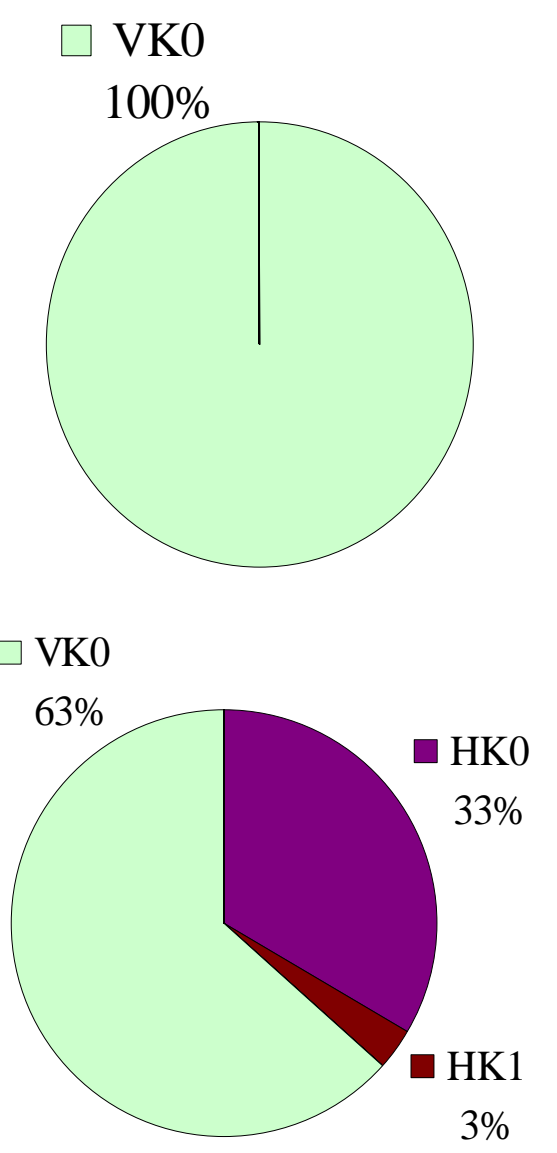

Abbildung 5. (A): Position der niedrigsten Schirmdämpfungswerte, Zertifizierter Schutzanzug ( $80 \mathrm{MHz}-1 \mathrm{GHz}),($ B): Position der niedrigsten Schirmdämpfungswerte, Zertifizierter Schutzanzug $(1 \mathrm{GHz}-4 \mathrm{GHz})$

1- Polarisation: $\mathrm{H}=$ horizontal, $\mathrm{V}=$ vertikal

2- Sondenposition: $\mathrm{T}=$ Thorax, $\mathrm{K}=\mathrm{Kopf}$

3- Messposition: $0=0^{\circ}$ gedreht, $1=60^{\circ}$ gedreht, $2=120^{\circ}$ gedreht, $3=180^{\circ}$ gedreht, $4=240^{\circ}$ gedreht, $5=300^{\circ}$ gedreht

Es ist demnach allgemein festzuhalten, dass die Verwendung eines Schirmgewebes höherer Schirmwirkung nicht zwangsläufig zu einer höheren Schirmdämpfung des gesamten Schutzanzuges führt.

Durch die höhere Leitfähigkeit des Innengewebes PCCS 2131 kommt es im Innern des Prototyps zu stärkeren Hohlraumresonanzen als im zertifizierten Schutzanzug. Dadurch bildet sich im Innern des Prototyps ein stark erhöhtes elektrisches Feld aus. Dies wirkt kontraproduktiv zur erhöhten Schirmdämpfung des Gewebes und führt somit bei dem zertifizierten Schutzanzug und dem verbesserten Prototyp zu gleichen Endergebnissen in der Schirmwirkung.

Ziel ist es außerdem, Erkenntnisse über das Schirmdämpfungsverhalten einzelner Teilbereiche der HFSchutzkleidung, insbesondere Bereiche mit Verbindungen und Verschlüssen, zu erlangen. Die Messungen werden daher statt der in DIN 32780-100 geforderten drei Mess-
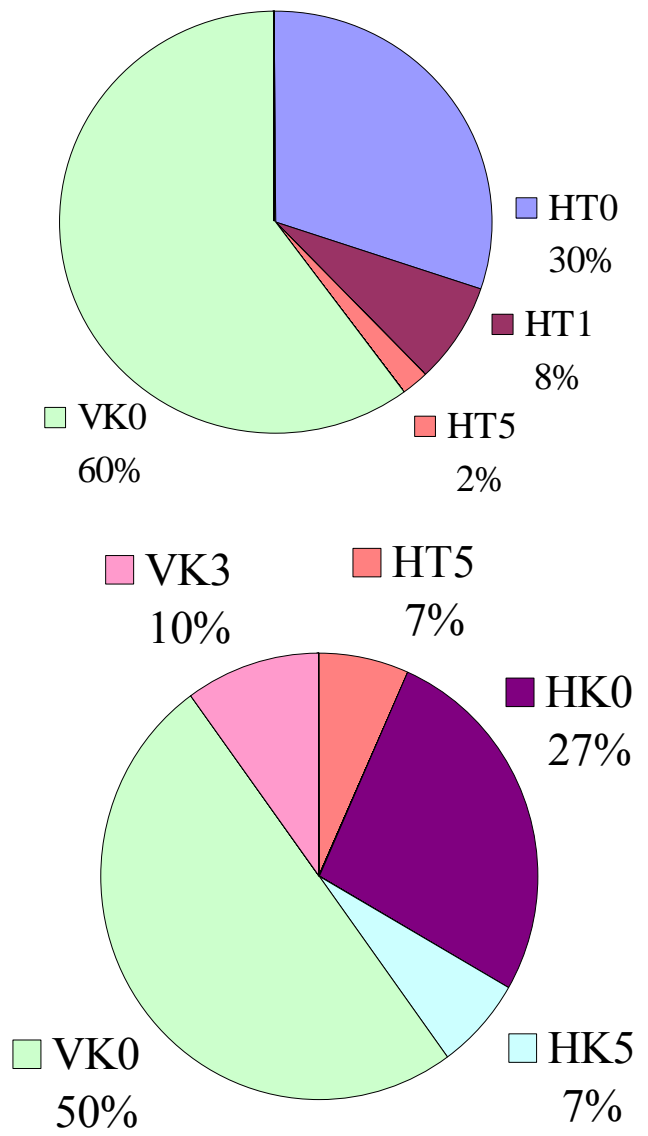

Abbildung 6. (A): Position der niedrigsten Schirmdämpfungswerte, Prototyp (80 MHz-1 GHz), (B): Position der niedrigsten Schirmdämpfungswerte, Prototyp ( $1 \mathrm{GHz}-4 \mathrm{GHz})$ Beschreibung der Positionen: siehe Bild 5.

positionen in sechs verschiedenen Positionen durchgeführt. Es wird daraufhin für jeden Frequenzschritt untersucht bei welcher Position der als Ergebnis angegebene niedrigste Schirmdämpfungswert auftritt.

Bilder 5 und 6 zeigen zu welchen Anteilen des untersuchten Frequenzbandes dieser niedrigste Wert bei den einzelnen Anordnungen auftrat. Es werden so die Schwachstellen der Schutzkleidung herausgearbeitet.

Bei dieser Untersuchung zeigt sich die Frontseite, also der mit Verschlüssen und Verbindungen versehene Bereich der Schutzkleidung, als Schwachstelle im Schirmdämpfungsverhalten. Als besonders kritisch zeigt sich der Kopfbereich mit dem integrierten Gesichtsgitter. Einzeluntersuchungen des Schirmdämpfungsverhaltens des verwendeten Gesichtsgitters haben jedoch ergeben, dass das Gitter selbst konstant gute Schirmdämpfungseigenschaften aufweist (siehe Bild 7).

Das Gesichtsgitter selbst kann somit als Schwachstelle ausgeschlossen werden. Die hier als Verknüpfung von Kopfhaube und Gesichtsgitter eingesetzten Reiß- und Klettverschlüsse verursachen demnach trotz ihres Metallanteils Unterbrechungen in der Leitfähigkeit. Somit ist kein 


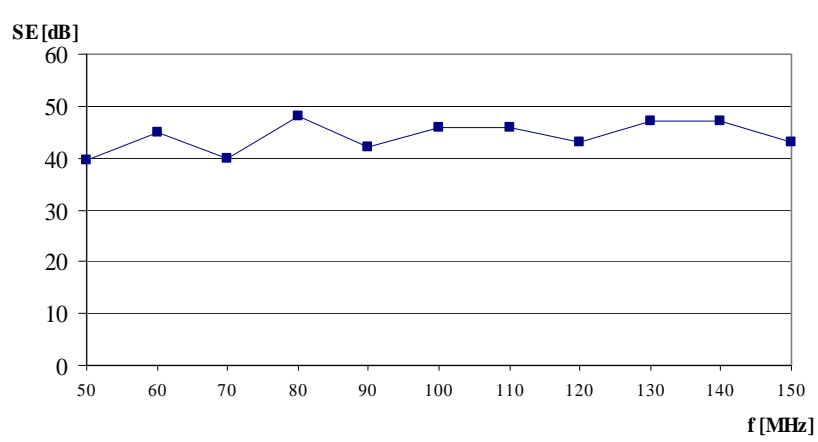

Abbildung 7. Schirmdämpfung des Gesichtsgitters.

ungehinderter Fluss des induzierten Stromes gewährleistet. Die Lücken, die bei der Verknüpfung von Gewebe und Reißbzw. Klettverschluss entstehen, sind größer als die Maschenweite des verwendeten Gewebes. Es kommt daher in diesem Bereich verstärkt zu einer Lochkopplung. Von der gesamten Frontseite erweist sich hierbei der Kopfbereich als besonders kritisch. Die um das Gesichtsgitter herumlaufende Verbindung stellt demnach ein größeres Problem dar als die vertikal eingesetzte Verbindung im Brustbereich des Anzuges. Dies ist darauf zurückzuführen, dass es sich bei der Verbindung zwischen Gesichtsgitter und Kopfhaube um einen geschlossenen Umlauf handelt.
Um die Schirmwirkung von Schutzkleidung weiter zu verbessern, sind diese Erkenntnisse über die Schwachstellen zu berücksichtigen. Es ist demnach sinnvoll sich auf die Verbesserung der leitenden Übergänge bei den Verschlüssen und Verbindungen zu konzentrieren. Die Entwicklung und Verwendung eines Gewebes mit höherer Schirmwirkung dagegen kann zurückgestellt werden.

\section{Literatur}

BGV B11: Unfallverhütungsvorschrift Elektromagnetische Felder, Berufsgenossenschaft der Feinmechanik und Elektrotechnik, 2001.

DIN 32780-100: Deutsche Norm Schutzkleidung, Teil 100: Schutz gegen hochfrequente elektromagnetische Felder im Frequenzbereich $80 \mathrm{MHz}$ bis $1 \mathrm{GHz}$, Anforderung und Prüfung, 2002.

Hummel, V. and Koch, M.: Schutzkleidung für den Personenschutz in hochfrequenten elektromagnetischen Feldern, Aktuelle Normung und Messverfahren, in: EMV 2004, herausgegeben von: Schwab, A., Tagungsband, VDE-Verlag, 2004.

Koch, M.: Applications of Electrically Conductive Textiles, 14th International Zurich Symposium in Electromagnetic Compatibility, Conference Proceedings, 2001. 